\title{
A Cell-Adhesive Plasma Polymerized Allylamine Coating Reduces the In Vivo Inflammatory Response Induced by Ti6A14V Modified with Plasma Immersion Ion Implantation of Copper
}

\author{
Uwe Walschus ${ }^{1}$, Andreas Hoene ${ }^{2}$, Maciej Patrzyk ${ }^{2}$, Silke Lucke ${ }^{1}$, Birgit Finke ${ }^{3}$, Martin Polak ${ }^{3}$, \\ Gerold Lukowski ${ }^{4}$, Rainer Bader ${ }^{5}$, Carmen Zietz ${ }^{5}$, Andreas Podbielski ${ }^{6}$, J. Barbara Nebe ${ }^{7}$ and \\ Michael Schlosser ${ }^{1, *}$ \\ 1 Research Group of Predictive Diagnostics, Department of Medical Biochemistry and Molecular Biology, \\ University Medical Center Greifswald, 17475 Greifswald, Germany; \\ uwe.walschus@uni-greifswald.de (U.W.); luckes@uni-greifswald.de (S.L.) \\ 2 Department of Surgery, University Medical Center Greifswald, 17475 Greifswald, Germany; \\ hoene@uni-greifswald.de (A.H.); patrzyk@uni-greifswald.de (M.Pa.) \\ 3 Leibniz Institute for Plasma Science and Technology (INP), 17489 Greifswald, Germany; \\ finke@inp-greifswald.de (B.F.); polak@inp-greifswald.de (M.Po.) \\ 4 Institute of Marine Biotechnology, 17489 Greifswald, Germany; lukowski@uni-greifswald.de \\ 5 Department of Orthopaedics, University Medical Center Rostock, 18057 Rostock, Germany; \\ rainer.bader@med.uni-rostock.de (R.B); carmen.zietz@med.uni-rostock.de (C.Z.) \\ 6 Department of Medical Microbiology, Virology and Hospital Hygiene, University Medical Center Rostock, \\ 18057 Rostock, Germany; andreas.podbielski@med.uni-rostock.de \\ 7 Department of Cell Biology, University Medical Center Rostock, 18057 Rostock, Germany; \\ barbara.nebe@med.uni-rostock.de \\ * Correspondence: schlosse@uni-greifswald.de; Tel.: +49-3834-8619-178
}

Received: 30 May 2017; Accepted: 17 July 2017; Published: 20 July 2017

\begin{abstract}
Copper $(\mathrm{Cu})$ could be suitable to create anti-infective implants based on Titanium (Ti), for example by incorporating $\mathrm{Cu}$ into the implant surface using plasma immersion ion implantation (Cu-PIII). The cytotoxicity of $\mathrm{Cu}$ might be circumvented by an additional cell-adhesive plasma polymerized allylamine film (PPAAm). Thus, this study aimed to examine in vivo local inflammatory reactions for Ti6Al4V implants treated with $\mathrm{Cu}-\mathrm{PIII}(\mathrm{Ti}-\mathrm{Cu})$, alone or with an additional PPAAm film (Ti-Cu-PPAAm), compared to untreated implants (Ti). Successful Cu-PIII and PPAAm treatment was confirmed with X-ray Photoelectron Spectroscopy. Storage of Ti-Cu and Ti-Cu-PPAAm samples in double-distilled water for five days revealed a reduction of $\mathrm{Cu}$ release by PPAAm. Subsequently, $\mathrm{Ti}$, Ti-Cu and Ti-Cu-PPAAm samples were simultaneously implanted into the neck musculature of 24 rats. After 7, 14 and 56 days, peri-implant tissue was retrieved from 8 rats/day for morphometric immunohistochemistry of different inflammatory cells. On day 56, Ti-Cu induced significantly stronger reactions compared to $\mathrm{Ti}$ (tissue macrophages, antigen-presenting cells, $\mathrm{T}$ lymphocytes) and to Ti-Cu-PPAAm (tissue macrophages, T lymphocytes, mast cells). The response for Ti-Cu-PPAAm was comparable with Ti. In conclusion, PPAAm reduced the inflammatory reactions caused by Cu-PIII. Combining both plasma processes could be useful to create antibacterial and tissue compatible Ti-based implants.
\end{abstract}

Keywords: titanium; copper; antibacterial properties; plasma polymerization; plasma immersion ion implantation; biocompatibility; morphometry; inflammatory response 


\section{Introduction}

Successful medical use of biomaterials requires a sufficient level of biocompatibility as well as specific physical and chemical properties suitable for the intended application. As established by experience of clinical use for bone replacement, titanium and its alloy Ti6Al4V (Ti) demonstrate good biocompatibility and excellent mechanical strength. While Ti based implants were found to fulfil their intended function for up to ten years after implantation and beyond [1], there is still potential for improvement. Among the most serious implant-related problems, bacterial infections due to the gram-positive species Staphylococcus aureus and Staphylococcus epidermidis are the primary cause of implant failure [2,3]. Additionally, it has been shown that the protein layer which is initially formed on Ti implants after implantation as part of the implantation-related host reactions renders the surface susceptible to bacterial colonization and the formation of bacterial biofilms [4].

Therefore, the modification of the surface of implants by different coatings to improve their resistance against infections, for example antibiotics, organic or inorganic antimicrobial agents, adhesion-resistant coatings, antibacterial bioactive polymers or nitrogen-monoxide delivering coatings, has been investigated [4]. As an alternative approach, we evaluated a low-temperature plasma-based surface treatment called plasma immersion ion implantation of copper (Cu-PIII), resulting in $\mathrm{Cu}$-releasing Ti surfaces with antibacterial properties as demonstrated by reduction of planktonic and biofilm-attached bacteria $[5,6]$. The underlying mechanism is the release of $\mathrm{Cu}$ ions which were implanted into the titanium oxide layer on top of the Ti surface. However, $\mathrm{Cu}$ is also toxic to mammal cells in a concentration-dependent manner, possibly causing adverse tissue effects in vivo. This was, for example, demonstrated in a study using Ti samples with a layer of galvanically deposited $\mathrm{Cu}$ which induced stronger acute inflammatory reactions than untreated control samples during the first three days following implantation in rats [7]. Therefore, such adverse effects should be minimized in order to reduce impacts in the peri-implant tissue while still maintaining the antibacterial properties.

This could be achieved by an additional layer with bioactive properties for modulation of tissue-surface interactions. In previous studies, we examined several surface treatments based on plasma polymerized allylamine (PPAAm), resulting in an amino-group rich, positively charged Ti surface characterized by robust anchoring of the PPAAm film with the Ti substrate due to the formation of carbide and oxycarbide bonds, as recently demonstrated by other authors [8]. We were able to show that these PPAAm surfaces had beneficial effects regarding rapid formation of osteoblastic focal adhesions of MG63 cells mediated by paxillin, vinculin and the phosphorylated focal adhesion kinase [9], and were also advantageous for cell morphology and spreading in vitro. Moreover, we were able to demonstrate in a recent in vivo study that, depending on the plasma process parameters, a reduced chronic local inflammatory response was obtained following implantation of PPAAm coated Ti plates in rats [10]. Furthermore, a study on Ti samples treated with a magnetron-sputtered mixed $\mathrm{Ti} / \mathrm{Cu}$ layer and an additional coating with plasma polymerized ethylenediamine, resulting in an amino-group rich positively charged surface similar to PPAAm, indicated that such cell-adhesive layers could diminish the inflammatory reactions induced by $\mathrm{Cu}$ [11]. Interestingly, microbiological experiments in one of our previous studies with Staphylococcus aureus cultivation on Cu-PIII-treated Ti samples, either without or with an additional PPAAm film, demonstrated that PPAAm moderately reduced the antibacterial activity of the surface but did not disable it [5]. Thus, an additional coating with PPAAm might be suitable to create a bioactive layer with beneficial effects on the surface of Cu-releasing Ti implants.

Of central relevance for the in vivo biocompatibility of an implant is the inflammatory response, influencing its short- and long-term stability and biofunctionality. Most important among the cells responsible for these reactions are macrophages and other phagocytic cells [12]. Furthermore, $\mathrm{T}$ lymphocytes and other immune cells are also involved in implantation-related host reactions [13], although their exact role has not been clarified so far [14,15]. Additionally, mast cells were found to mediate the acute inflammatory response after implantation [16], and recent work demonstrated 
the infiltration of natural killer (NK) cells in the context of particle-mediated periprosthetic inflammation [17].

Therefore, the aim of this study was to examine the short- and long-term inflammatory in vivo reactions after simultaneous implantation of Ti plates with either a Cu-PIII treatment alone (Ti-Cu) or a combination of a Cu-PIII treatment and an additional PPAAm layer (Ti-Cu-PPAAm) in comparison to untreated Ti control samples in rats. For this, the evaluation of the local inflammatory response by total monocytes/macrophages, tissue macrophages, $\mathrm{T}$ lymphocytes, $\mathrm{MHC}-\mathrm{II}^{+}$antigen-presenting cells, mast cells and activated NK cells in the peri-implant tissue were morphometrically determined by immunohistochemistry and digital image analysis.

\section{Results}

\subsection{Physico-Chemical Surface Analysis}

The results from ex-situ X-ray photoelectron spectroscopy (XPS) elemental surface analysis, shown in Table 1, demonstrate a comparable elemental composition for Ti-Cu samples and Ti controls (Ti-6Al-4V) regarding the titanium content (Ti-Cu: $12.7 \%$ vs. Ti: $16.3 \%)$, the carbon content (Ti-Cu: $30.2 \%$ vs. Ti: $32.4 \%$ ), and the oxygen content (Ti-Cu: $45.7 \%$ vs. Ti: $44.4 \%$ ). In contrast, copper was only detected for the Ti-Cu samples with a relative amount of $4.1 \%$. On the other hand, the Ti-Cu-PPAAm samples had a markedly higher content of carbon $(74.5 \%)$ and nitrogen $(23.2 \%)$ than the Ti-Cu samples and the Ti controls. Neither titanium nor copper and only 2.2\% oxygen were found on the surface of Ti-Cu-PPAAm samples.

Table 1. Elemental surface composition of unmodified Ti6Al4V plates (Ti), Ti6Al4V plates treated with plasma immersion ion implantation of copper $(\mathrm{Ti}-\mathrm{Cu})$ or Ti6Al4V plates treated with plasma immersion ion implantation of copper and an additional plasma polymerized allylamine film (Ti-Cu-PPAAm) determined by X-ray photoelectron spectroscopy (XPS); data are given as mean and standard deviation of measurements at $n=3$ different surface positions of the same sample.

\begin{tabular}{cccccccc}
\hline Implant Series & Ti [\%] & Al [\%] & V [\%] & Cu [\%] & C [\%] & O [\%] & N [\%] \\
\hline $\mathrm{Ti}$ & $16.3 \pm 0.2$ & $2.5 \pm 0.4$ & $0.4 \pm 0.1$ & 0 & $32.4 \pm 0.9$ & $44.4 \pm 0.9$ & $2.7 \pm 0.3$ \\
$\mathrm{Ti}-\mathrm{Cu}$ & $12.7 \pm 0.2$ & $0.7 \pm 0.04$ & $0.4 \pm 0.03$ & $4.1 \pm 0.1$ & $30.2 \pm 1.3$ & $45.7 \pm 1.3$ & 0 \\
Ti-Cu-PPAAm & 0 & 0 & 0 & 0 & $74.5 \pm 0.3$ & $2.2 \pm 0.1$ & $23.2 \pm 0.2$ \\
\hline
\end{tabular}

Furthermore, the XPS depth profile was analyzed for Ti-Cu samples. Changes of the elemental composition induced by Cu-PIII could be characterized up to a depth of about $45 \mathrm{~nm}$ (Figure 1). $\mathrm{Cu}$ was implanted underneath the surface, with two maxima for the $\mathrm{Cu}$ content at depths of $1.5 \mathrm{~nm}$, i.e., directly on surface level, and around $15 \mathrm{~nm}$, respectively.

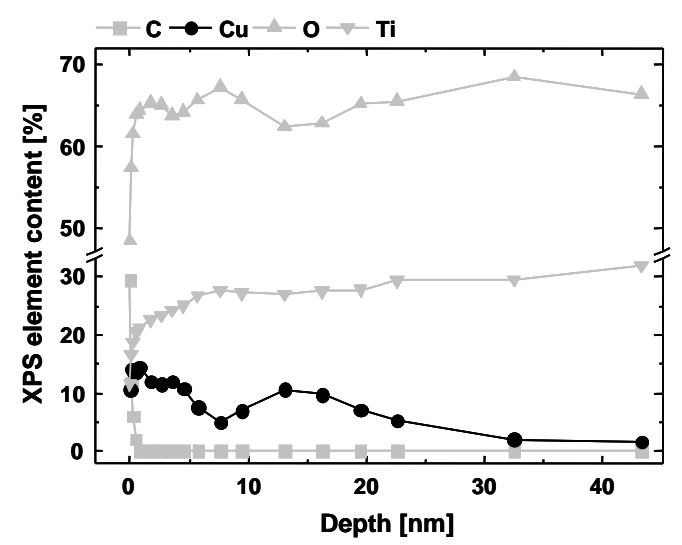

Figure 1. X-ray photoelectron depth profile analysis of a polished Ti6Al4V plate surface treated with plasma immersion ion implantation of copper $(\mathrm{Ti}-\mathrm{Cu})$. 


\subsection{Analysis of Copper Release}

Cumulative $\mathrm{Cu}$ concentrations released from Ti-Cu and Ti-Cu-PPAAm samples in $2 \mathrm{~mL}$ double-distilled water over time are summarized in Figure 2. After $24 \mathrm{~h}$, the concentration was 0.0022 $\pm 0.0002 \mathrm{mmol} \mathrm{L}^{-1}$ for Ti-Cu, about twice as much as for Ti-Cu-PPAAm $\left(0.001 \pm 0.0002 \mathrm{mmol} \mathrm{L}^{-1}\right)$. The difference between both sample series after 5 days was $0.0086 \pm 0.0022 \mathrm{mmol} \mathrm{L}^{-1}$ for Ti-Cu vs. $0.0025 \pm 0.0004 \mathrm{mmol} \mathrm{L}^{-1}$ for Ti-Cu-PPAAm.

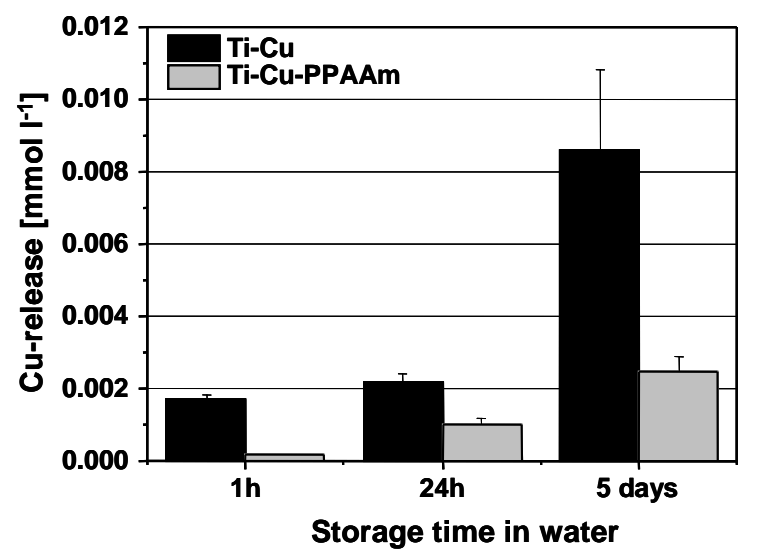

Figure 2. $\mathrm{Cu}$ concentrations released from Ti6Al4V plates treated with plasma immersion ion implantation of copper ( $\mathrm{Ti}-\mathrm{Cu}$, dark bars) or Ti6Al4V plates treated with plasma immersion ion implantation of copper and an additional plasma polymerized allylamine film (Ti-Cu-PPAAm, gray bars) in $2 \mathrm{~mL}$ double distilled water over time. Bars represent the mean and whiskers the standard deviation of $n=3$ different samples for each series.

\subsection{Morphological Examination}

\subsection{1. $\mathrm{CD}^{+}{ }^{+}$Total Monocytes/Macrophages (ED1)}

For the number of $\mathrm{CD}^{+} 8^{+}$total monocytes/macrophages (Figure 3a), a significant decrease was

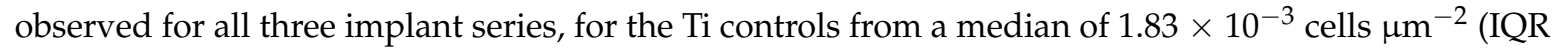
$\left.1.58 \times 10^{-3}-1.93 \times 10^{-3}\right)$ on day 7 to $0.95 \times 10^{-3}$ cells $\mu \mathrm{m}^{-2}\left(\mathrm{IQR} 0.63 \times 10^{-3}-1.09 \times 10^{-3}\right)$ on day $56(p=0.0060)$, for Ti-Cu from a median of $1.77 \times 10^{-3}\left(\right.$ IQR $\left.1.46 \times 10^{-3}-2.09 \times 10^{-3}\right)$ on day 7 to $0.98 \times 10^{-3}\left(\right.$ IQR $\left.0.83 \times 10^{-3}-1.13 \times 10^{-3}\right)$ on day $56(p=0.0007)$, and for Ti-Cu-PPAAm from a median of $1.86 \times 10^{-3}\left(\right.$ IQR $\left.1.50 \times 10^{-3}-2.10 \times 10^{-3}\right)$ on day 7 to $1.03 \times 10^{-3}\left(\right.$ IQR $0.49 \times 10^{-3}-1.21 \times 10^{-3}$ ) on day $56(p=0.0009)$. There was no significant difference between the implant series on any experimental day.

\subsection{2. $\mathrm{CD}_{163^{+}}$Tissue Macrophages (ED2)}

Similar to the $\mathrm{CD} 68^{+}$total monocytes/macrophages, the number of $\mathrm{CD} 163^{+}$tissue macrophages (Figure 3b) decreased significantly for all three implant series, for the Ti controls from a median of $1.33 \times 10^{-3}\left(\right.$ IQR $\left.1.02 \times 10^{-3}-1.57 \times 10^{-3}\right)$ on day 7 to $0.57 \times 10^{-3}\left(\right.$ IQR $\left.0.48 \times 10^{-3}-0.74 \times 10^{-3}\right)$ on day $56(p=0.0072)$, for Ti-Cu from a median of $1.34 \times 10^{-3}\left(\right.$ IQR $\left.0.96 \times 10^{-3}-1.57 \times 10^{-3}\right)$ on day 7 to $0.78 \times 10^{-3}\left(\right.$ IQR $\left.0.60 \times 10^{-3}-0.87 \times 10^{-3}\right)$ on day $56(p=0.01)$, and for Ti-Cu-PPAAm from a median of $1.58 \times 10^{-3}\left(\right.$ IQR $1.33 \times 10^{-3}-1.73 \times 10^{-3}$ ) on day 7 to $0.48 \times 10^{-3}\left(\right.$ IQR $\left.0.39 \times 10^{-3}-0.58 \times 10^{-3}\right)$ on day $56(p=0.0003)$. While no differences were found between the implant series on experimental days 7 and 14, the Ti-Cu implants had a significantly higher number of $\mathrm{CD} 163^{+}$tissue macrophages compared to both the Ti-Cu-PPAAm implants and the Ti controls on day 56 ( $p=0.0078$ respectively). Furthermore, the number of $\mathrm{CD} 163^{+}$tissue macrophages for the Ti-Cu-PPAAm implants was lower than for the Ti controls on day $56(p=0.0781)$. 


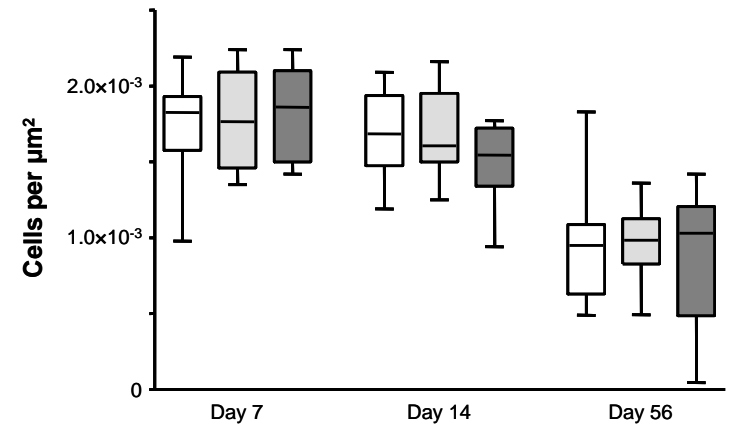

(a)
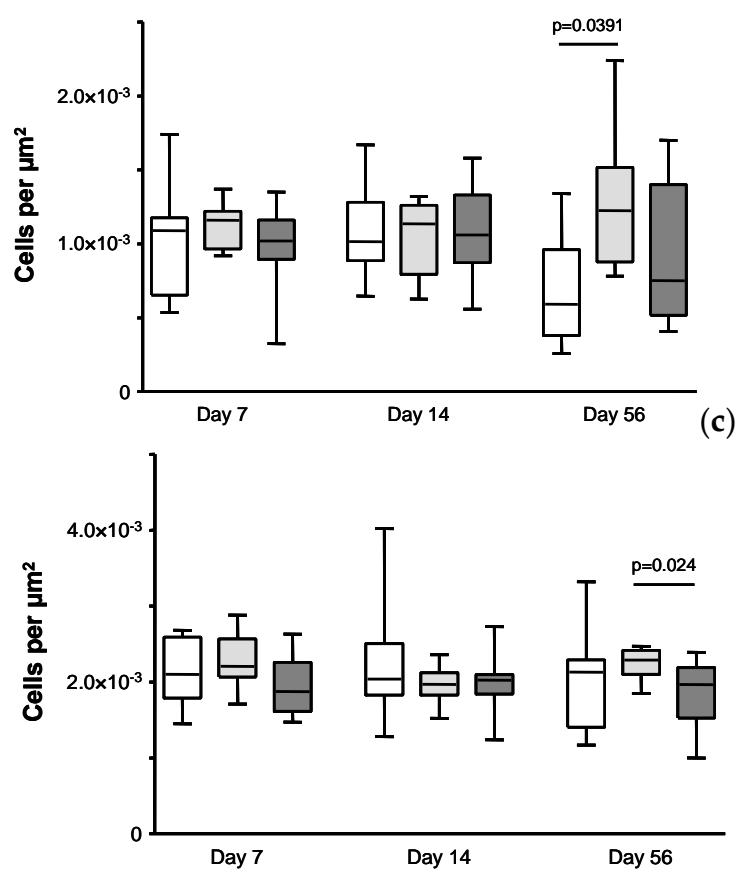

(e)

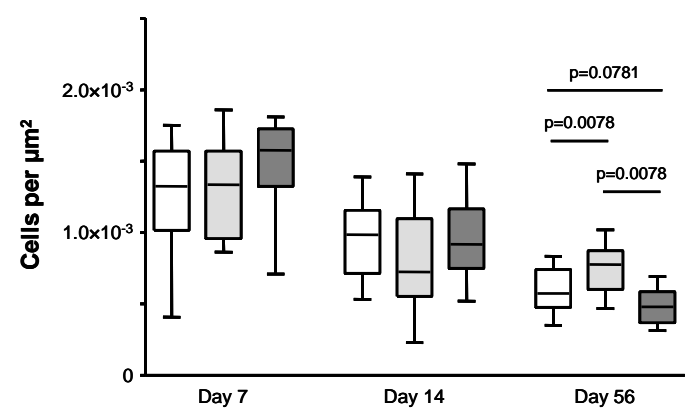

(b)

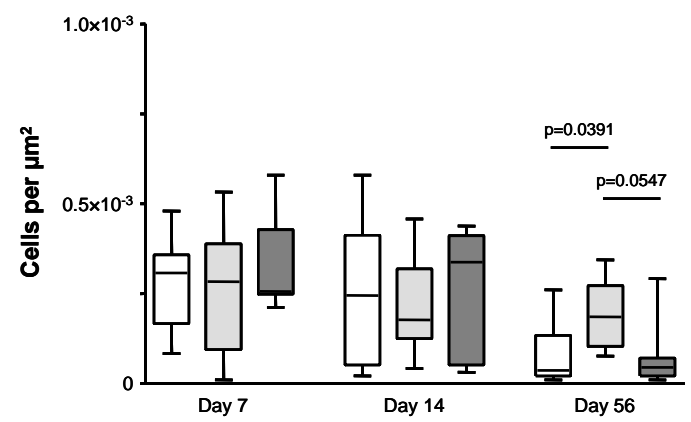

(d)

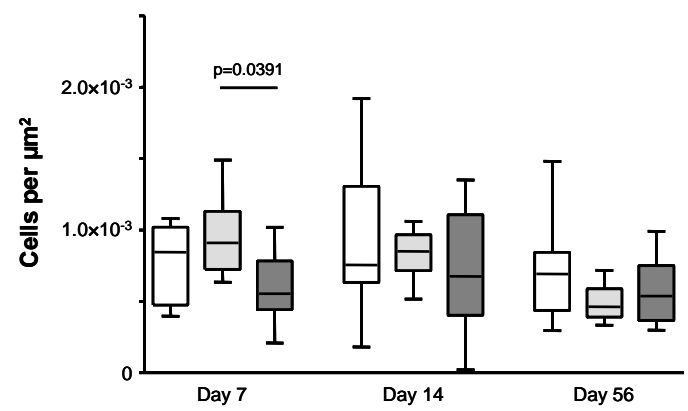

(f)

Figure 3. Number of (a) $\mathrm{CD}^{+} 8^{+}$total monocytes and pro-inflammatory macrophages; (b) CD163 ${ }^{+}$ anti-inflammatory tissue macrophages; (c) MHC-II ${ }^{+}$antigen-presenting cells; (d) total T lymphocytes; (e) mast cells; and (f) activated natural killer cells in the peri-implant tissue of Lewis rats after i.m. implantation of unmodified Ti6Al4V plates (Ti, white boxes), Ti6Al4V plates treated with plasma immersion ion implantation of copper ( $\mathrm{Ti}-\mathrm{Cu}$, light gray boxes) or Ti6Al4V plates treated with plasma immersion ion implantation of copper and an additional plasma polymerized allylamine film (Ti-Cu-PPAAm, dark gray boxes) after 7,14 and 56 days. Boxes represent median and interquartile range and whiskers minimum and maximum values; $p$-values indicate differences in pairwise comparison by non-parametric Wilcoxon signed rank test.

\subsubsection{MHC-II ${ }^{+}$Antigen-Presenting Cells (OX6)}

For the number of MHC-II+ antigen-presenting cells (Figure 3c), no change over time was observed for any of the three implant series. On experimental days 7 and 14, no differences between the implant series were found. However, on day 56 the Ti-Cu implants had significantly higher numbers than the Ti controls (median $1.23 \times 10^{-3}$, IQR $0.88 \times 10^{-3}-1.52 \times 10^{-3}$ vs. median $0.59 \times 10^{-3}$, IQR $\left.0.38 \times 10^{-3}-0.96 \times 10^{-3} ; p=0.0391\right)$. 


\subsubsection{T Lymphocytes (R73)}

The number of $\mathrm{T}$ lymphocytes (Figure 3d) decreased significantly from day 7 to day 56 for the Ti controls, from a median of $0.31 \times 10^{-3}$ (IQR $0.17 \times 10^{-3}-0.36 \times 10^{-3}$ ) on day 7 to $0.04 \times 10^{-3}$ (IQR $\left.0.02 \times 10^{-3}-0.13 \times 10^{-3}\right)$ on day $56(p=0.0146)$ and for the Ti-Cu-PPAAm implants, from a median of $0.27 \times 10^{-3}\left(\right.$ IQR $\left.0.25 \times 10^{-3}-0.43 \times 10^{-3}\right)$ on day 7 to $0.05 \times 10^{-3}\left(\right.$ IQR $\left.0.02 \times 10^{-3}-0.07 \times 10^{-3}\right)$ on day $56(p=0.0175)$, but not for the Ti-Cu implants. Consequently, the Ti-Cu implants had significantly higher numbers of T lymphocytes on day 56 (median $0.18 \times 10^{-3}$, IQR $0.10 \times 10^{-3}-0.27 \times 10^{-3}$ ) in comparison to the Ti controls $(p=0.0391)$ and to the Ti-Cu-PPAAm implants $(p=0.05)$, while there was no significant difference between the three implant series on days 7 or 14 .

\subsubsection{Mast Cells (AD1)}

The numbers of mast cells (Figure 3e) remained unchanged over the course of the study for all three implant series. On day 56, the Ti-Cu implants had significantly higher numbers of mast cells in comparison to the Ti-Cu-PPAAm implants (median $2.29 \times 10^{-3}$, IQR $2.10 \times 10^{-3}-2.42 \times 10^{-3} \mathrm{vs}$. median $1.97 \times 10^{-3}$, IQR $\left.1.53 \times 10^{-3}-2.19 \times 10^{-3} ; p=0.024\right)$.

\subsubsection{Activated NK Cells (ANK61)}

For the numbers of activated NK cells (Figure 3f), a significant decrease was observed only for the Ti-Cu implants, from a median of $0.91 \times 10^{-3}$ (IQR $0.72 \times 10^{-3}-1.13 \times 10^{-3}$ ) on day 7 to $0.46 \times 10^{-3}\left(\right.$ IQR $\left.0.39 \times 10^{-3}-0.59 \times 10^{-3}\right)$ on day $56(p=0.0016)$, but not for the other two implant series. This was mainly due to an elevated number of activated NK cells for the Ti-Cu implants in the early phase, significantly higher than for the Ti-Cu-PPAAm implants with a median of $0.55 \times 10^{-3}$ (IQR $0.44 \times 10^{-3}-0.78 \times 10^{-3}$ ) on day $7(p=0.024)$, but also with a lesser extent compared to the Ti controls. On days 14 and 56, no differences were found between the three implant series.

\section{Discussion}

Anti-infective biomaterials could improve the clinical outcome following implantation since bacterial infections are a leading cause for implant failure. Previous in vitro studies showed antibacterial properties for Ti6Al4V (Ti) surfaces either treated with plasma immersion ion implantation of copper (Cu-PIII) alone or with Cu-PIII followed by an additional plasma polymerized allylamine (PPAAm) film [5,6]. Earlier experiments also demonstrated that PPAAm is advantageous regarding rapid focal adhesion formation, morphology and the spreading of MG63 cells in vitro [9], with no negative influence on the local tissue reactions and a reduced chronic inflammatory response in vivo [10]. Consequently, the present study aimed at evaluating the in vivo effects of an additional PPAAm film on Cu-PIII-treated Ti plates regarding the acute and chronic local inflammatory reactions.

XPS demonstrated that Ti-Cu samples were overall comparable to Ti controls for relative amounts of titanium, carbon, oxygen and nitrogen. XPS also confirmed $\mathrm{Cu}$ incorporation by $\mathrm{Cu}$-PIII into the $\mathrm{TiO}_{2}$ layer and $\mathrm{Ti}$ itself. In comparison, Ti-Cu-PPAAm samples had higher amounts of carbon and nitrogen as PPAAm films consist of a cross-linked plasma-polymer nanolayer with a hydrocarbon network containing nitrogen functional groups such as amines, acid amides, imines and nitriles [18]. Furthermore, neither Ti nor $\mathrm{Cu}$ and only a small oxygen amount were detected on Ti-Cu-PPAAm samples, indicating complete coverage by PPAAm with a thickness of about $50 \mathrm{~nm}$. The XPS depth profile revealed two peaks for the $\mathrm{Cu}$ distribution, one at a depth of about $1.5 \mathrm{~nm}$ and a second around $15 \mathrm{~nm}$. The first peak is probably due to coating processes with low energetic $\mathrm{Cu}$ ions, while the second peak is the actual implantation peak. However, it should be noted that the measured depth is slightly too high for implantation of $10 \mathrm{keV} \mathrm{Cu}$ ions into Ti as computer simulations with the software system TRIM 2013 ('Transport of Ions in Matter'; (C) 1984-2013, James F. Ziegler) gave a simulated mean depth of about $10 \mathrm{~nm}$. The difference can be mainly explained by two effects, on the one hand the $\mathrm{Cu}$ coating on top of the surface and on the other hand by the measuring method itself (XPS with depth 
profiling). The Ar ions used for depth profiling have an energy of $4.5 \mathrm{keV}$. They not only induce surface sputtering but also hit the implanted $\mathrm{Cu}$ atoms inside the titanium, thereby driving them deeper into the substrate. Furthermore, the photoelectrons from the surface measured with XPS are an integration over the depth of up to $5 \mathrm{~nm}$ (mean free path of electrons in titanium).

Subsequent examination of the $\mathrm{Cu}$ release from $\mathrm{Ti}-\mathrm{Cu}$ and Ti-Cu-PAAAm samples however indicated that PPAAm inhibits the $\mathrm{Cu}^{2+}$ diffusion process to a certain degree. Moreover, released $\mathrm{Cu}^{2+}$ concentrations from the polished Ti surfaces used in the present study were also lower than, for example, for rougher corundum blasted surfaces treated by $\mathrm{Cu}$-PIII or dual high power impulse magnetron sputtering (dual HiPIMS) [19]. Furthermore, the $\mathrm{Cu}$ release results are consistent with earlier microbiological experiments in which both Ti-Cu and Ti-Cu-PPAAm samples reduced residual Staphylococcus aureus numbers by at least one order of magnitude after $24 \mathrm{~h}$ in contrast to Ti controls, with a moderate reduction of antibacterial effectiveness due to the additional PPAAm film [5]. By comparison, samples coated with $\mathrm{Cu}$ by dual HiPIMS had a stronger antimicrobial effect than Ti-Cu samples as used in the present study, which also showed a reduced cytotoxicity to MG-63 osteoblasts compared to the dual HiPIMS samples [19]. This underlines the importance of optimizing the $\mathrm{Cu}$ release kinetics regarding the balance between antibacterial and cytotoxic effects by choosing a suitable plasma treatment with appropriate process conditions.

Following physico-chemical evaluation, simultaneous intramuscular implantation of Ti-Cu, Ti-Cu-PPAAm and Ti control samples into the neck musculature of rats was performed for comparative in vivo examination of their effects on acute and chronic local inflammatory reactions. This experimental model, resembling the clinical situation of a predominantly muscular environment for orthopaedic Ti implants, was established and optimized in several recent studies $[7,10,11,20,21]$. It can be performed with minimal surgical complications and post-operative stress, which are relevant considerations for animal welfare. Furthermore, musculature is well supplied with blood and thus particularly suitable to study inflammatory tissue reactions. Simultaneous implantation of different samples into the same experimental animals also facilitates an intra-individual comparison, reducing both the number of animals required as well as the broad individual variability regarding inflammatory and immunological reactions as observed in earlier studies [22,23].

Overall, the histological results indicate a shift from acute to chronic inflammation for all three sample series. This is especially demonstrated by the decrease over time for numbers of $\mathrm{CD} 68^{+}$total monocytes/pro-inflammatory macrophages (ED1) and anti-inflammatory CD163 ${ }^{+}$tissue macrophages (ED2) as macrophages play a major role in biomaterial-related inflammation [12]. In the early acute phase of inflammation at day 7 the Ti-Cu implants induced the strongest reaction only for the activated NK cells, which possess no antigen-specific receptors and are part of the innate immune response. In the late chronic phase of inflammation at day 56, tissue reactions were strongest for Ti-Cu samples for the $\mathrm{CD}_{163}{ }^{+}$tissue macrophages, the MHC-II ${ }^{+}$antigen-presenting cells, the Tlymphocytes and the mast cells. These late phase observations, not seen for $\mathrm{CD} 68^{+}$total monocytes/pro-inflammatory macrophages and activated NK cells, possibly indicate a stronger involvement of immunological reactions induced by Ti-Cu implants in the chronic inflammation. Furthermore these results clearly demonstrate beneficial effects from an additional PPAAm film by reducing the long-term chronic inflammatory tissue response induced by $\mathrm{Cu}$-PIII. This is consistent with earlier results regarding another amino-group rich coating, plasma polymerized ethylendiamine (PPEDA), on the inflammatory reactions caused by Ti samples with a magnetron-sputtered $\mathrm{Cu}$ layer [11]. It should be noted that the $\mathrm{Cu}$-PIII treatment results in implantation of $\mathrm{Cu}$ into both the $\mathrm{TiO}_{2}$ layer and Ti itself while a mixed layer of atomic $\mathrm{Cu}$ and $\mathrm{Ti}$ is deposited onto the sample surface by dual HiPIMS as used in that recently published study [19]. Furthermore, the precursor ethylenediamine used for PPEDA coating has two amino groups versus one amino group for allylamine used for PPAAm coating, resulting in a different amino-group density. Another study using Ti samples treated with plasmaelectrolytical oxidation followed by galvanic $\mathrm{Cu}$ deposition revealed increased inflammatory reactions for $\mathrm{Cu}$-treated samples compared to controls already during the acute phase [7]. Thus, despite the different $\mathrm{Cu}$ deposition 
methods used in these studies and corresponding physico-chemical differences between the resulting samples, as well as between the cell-adhesive films PPAAm and PPEDA, the overall effects on the inflammatory tissue response were notably comparable.

In summary, an additional PPAAm film reduced the chronic local inflammatory reactions caused by Cu-PIII-treated Ti implants. This observation extends earlier in vitro results concerning their antibacterial activity $[5,6]$ as well as in vitro and in vivo studies on PPAAm as single cell-adhesive coating for Ti implants [9,10] and electronspun poly(L-lactide-co-D/L-lactide) meshes [24]. The combination of plasma immersion ion implantation of copper and plasma polymerized allylamine could thus be used for implants with both antibacterial and tissue compatible properties, aimed at preventing short-term infections while maintaining optimal tissue integration. However, the results also demonstrate that further studies are needed to find the optimal adjustment between these two aspects. Such studies could include the examination of additional aspects of the tissue response and cellular behavior aimed at providing a deeper understanding of the inflammatory response beyond the comparison between different surface modifications in the present study. Possible parameters include for example the expression of immune phenotype markers, the levels of reactive oxygen species (ROS), a differentiation of the $\mathrm{T}$ cell response into subpopulations such as helper $\mathrm{T}$ cells, cytotoxic $\mathrm{T}$ cells, or regulatory suppressor $\mathrm{T}$ cells, a closer look at pro- and anti-inflammatory markers like expression of different cytokines, or a Masson's trichrome staining to examine the extent of fibrosis. Such investigations could provide information on how different aspects of the short- and long-term inflammatory response on the one hand affect the implant performance and on the other hand are influenced by certain material properties, revealing important information regarding cell-material interactions and structure-property-function relationships which might also be relevant for other materials or surface modifications.

More comprehensive studies are also needed to fully assess the extent and duration of the antibacterial effects of samples treated with $\mathrm{Cu}-\mathrm{PIII}$ and PPAAm. The respective data in our previous study [5] covers only a short period of incubation with a small inoculation volume, which did not fully cover the samples. This experimental approach primarily had the short phase immediately after implantation in mind, which is often described as a 'race for the surface' between host cells and bacteria [25]. During this short period, impeding initial bacterial adhesion and colonization is especially important to prevent formation of a biofilm which is nearly impossible to remove in clinical practice. Additional experiments including extended incubation periods under liquid and flow-based conditions to better mimic the in vivo microenvironment are necessary to examine whether the antibacterial effectiveness corresponds to the in vitro release data and to address the long-term effects of the Cu-PIII/ PPAAm treatment on adherence, survival and biofilm formation of bacteria as well as their possible adjustment and selection. Furthermore, in vivo experiments using implants with spiked bacterial loads might help to evaluate the in vivo efficacy as well as the influence of bacterial infections on the tissue response, like the extent of fibrosis.

\section{Materials and Methods}

\subsection{Samples and Plasma Treatment}

\subsubsection{Samples}

Plasma treatments were carried out on either polished Ti6Al4V discs (diameter $11 \mathrm{~mm}$, height $1 \mathrm{~mm}$ ) for physicochemical surface analyses or small square Ti6Al4V platelets $\left(5 \times 5 \times 1 \mathrm{~mm}^{3}\right)$ for in vivo investigations, with a defined arithmetic roughness of $R_{a}=0.19 \mu \mathrm{m}$ or $R_{a}=0.28 \mu \mathrm{m}$ respectively (all samples obtained from DOT GmbH, Rostock, Germany). 


\subsubsection{Plasma Immersion Ion Implantation of Copper (Cu-PIII)}

Plasma Immersion Ion Implantation of copper (Cu-PIII) was performed in an ultra-high vacuum (UHV) reactor (constructed in-house at the Leibniz Institute for Plasma Science and Technology, Greifswald, Germany) with a capacitively coupled radio frequency-discharge (13.56 MHz) and two coplanar copper electrodes. The pulsed voltage applied was at $10 \mathrm{kV}$ and the working pressure at $2 \mathrm{~Pa}$. The pulses for the $\mathrm{Cu}-\mathrm{PIII}$ had a short rise time in the range of $200 \mathrm{~ns}$, a repetition rate of $1 \mathrm{kHz}$ and pulse length of $2 \mu \mathrm{s}$. These parameters induced a mean implantation current of about $7 \mathrm{~mA}$. The temperature of the sample attains about $<50{ }^{\circ} \mathrm{C}$. The $\mathrm{Cu}$-PIII treatment was carried out on each sample side for $55 \mathrm{~min}$. The resulting samples are named Ti-Cu.

\subsubsection{Creation of Plasma Polymerized Allylamine Films on Ti-Cu Surfaces (Ti-Cu-PPAAm)}

On one series of Ti-Cu samples, an additional plasma polymerized allylamine (PPAAm) thin film was deposited in a microwave (MW, $2.45 \mathrm{GHz}$ ) plasma reactor V55G (Plasma-finish GmbH, Schwedt/Oder, Germany) in a two-step procedure without breaking the vacuum. At first, all samples were decontaminated and activated by a continuous wave oxygen/argon plasma (500 W, $50 \mathrm{~Pa}, 100 \mathrm{sccm}$ $\mathrm{O}_{2} / 25 \mathrm{sccm}$ Ar) and secondly followed by the deposition of the PPAAm thin film ( $50 \mathrm{~nm}$ thick) ( $500 \mathrm{~W}, 50 \mathrm{~Pa}, 50 \mathrm{sccm}$ allylamine/50 sccm Ar; pulsed plasma regime $0.3 \mathrm{~s}$ on/1.7 s off). The overall plasma on time was $144 \mathrm{~s}$ and the total processing time $960 \mathrm{~s}$. The PPAAm plasma deposition process was performed on each sample side. The resulting samples are named Ti-Cu-PPAAm.

\subsection{Physico-Chemical Analysis}

\subsubsection{X-ray Photoelectron Spectroscopy (XPS) Analysis}

The elemental chemical surface composition and chemical binding properties of the different implant series were examined by XPS with an AXIS ULTRA spectrometer (Kratos, Manchester, UK) as previously described in detail [5]. In summary, the monochromatic $\mathrm{Al} \mathrm{K} \alpha$ line at $1486 \mathrm{eV}(150 \mathrm{~W})$ was used with implemented charge neutralization and a pass energy of $80 \mathrm{eV}$ for estimating the chemical elemental composition or of $10 \mathrm{eV}$ for highly resolved peaks. The C-C/C-H component of the $\mathrm{C} 1 \mathrm{~s}$ peak was adjusted to $285 \mathrm{eV}$ [26]. Each measurement was repeated three times at different surface positions of the same sample.

XPS depth profiles were recorded by sputtering with $\mathrm{Ar}^{+}$ions with a kinetic energy of $4.5 \mathrm{keV}$ for a specific time. After a certain sputtering time, the ion flux was interrupted and the elemental composition of the actual surface was determined. Afterwards the next sputtering cycle was performed. The software system CasaXPS version 2.3.15 (Casa Software Ltd, Teignmouth, UK) was used for quantification.

\subsubsection{Measurement of PPAAm Film Thickness}

Measurement of PPAAm film thickness was performed using the surface profiler Dektak 3ST (Veeco, Plainview, NY, USA) as described previously [9]. Briefly, the silica wafer was partially coated before plasma treatment with a cellulose acetate film which was removed after PPAAm film deposition, taking along the plasma polymer situated thereon. The film thickness was subsequently determined with the surface profiler (stylus tip radius: $2.5 \mathrm{~mm}$ ) as the level difference of the step created by removal of the cellulose acetate film, based on the mean of 25 measurement points on each side of the step.

\subsubsection{Copper Release Measurement}

The copper release from Ti-Cu and Ti-Cu-PPAAm samples was measured by storing the samples in $2 \mathrm{~mL}$ double-distilled water (TKA Wasseraufbereitungssysteme $\mathrm{GmbH}$, Niederelbert, Germany) for five days at room temperature. $\mathrm{Cu}$ concentrations in storage liquid were measured by means of atomic 
absorption spectrometry (AAS) ZEEnit 650 (Analytik Jena AG, Jena, Germany) with electro-thermal atomisation. Three separate samples from each series were analyzed.

\subsection{In Vivo Experiments}

\subsubsection{Laboratory Animals}

24 male Lewis rats (age 100 days, mean weight $356 \pm 11 \mathrm{~g}$ ) were kept in in-house facilities of the University Medical Center Greifswald under conventional housing and feeding conditions. All animal experiments were performed in accordance with the animal protection law of the Federal Republic of Germany in its new version of 1 January 1987, with the principles of care for animals in laboratories (drawn up by the National Society for Medical Research) and with the Guidelines for Keeping and Using Laboratory Animals (NIH Publication No.80-23, revised 1985). The study was reviewed and approved (approval code 7221.3-1.1-074/11) by the State Office for Agriculture, Food Safety and Fishery of the federal state of Mecklenburg-Vorpommern (Rostock, Germany).

\subsubsection{Implantation Procedure and Tissue Sampling}

The animals were anesthetized by i.p. application of a mixture of Rompun ${ }^{\circledR}$ (Bayer, Leverkusen, Germany) and Ketamin ${ }^{\circledR}$ (Sanofi-Ceva, Düsseldorf, Germany) with dosing relative to individual body weight. Three implant samples were simultaneously implanted in each animal into small intramuscular pockets in the neck musculature: one implant treated with plasma immersion ion implantation of copper only ( $\mathrm{Ti}-\mathrm{Cu})$, one implant treated with plasma immersion ion implantation of copper and an additional plasma polymerized allylamine coating (Ti-Cu-PPAAm) and one untreated Ti6Al4V control implant (Ti). The three implants were separated by at least $2 \mathrm{~cm}$ from each other in a triangular alignment and fixed in their respective tissue pockets with a nonresorbable synthetic polypropylene suture (PROLENE ${ }^{\circledR}$; Ethicon Endo-Surgery, Inc., Hamburg, Germany).

Eight randomly selected animals were euthanized after 7, 14 and 56 days, and the implants with a sample of the surrounding tissue were carefully explanted after surgical opening of the implantation site. After immediate freezing of the samples with laboratory freezer spray New Envi-Ro-Tech ${ }^{\mathrm{TM}}$ (Thermo Electron Corporation, Pittsburgh, PA, USA), they were cut with a scalpel with the section plane at right angle with the implants after which the implants were carefully removed from the frozen tissue using tweezers. The embedding medium Shandon Cryomatrix ${ }^{\mathrm{TM}}$ (Thermo Electron Corporation, Pittsburgh, PA, USA) was used to fill the remaining tissue pockets to preserve their form during further processing. The tissue samples were subsequently shock frozen and stored at $-80^{\circ} \mathrm{C}$.

\subsection{Morphological Examination}

\subsubsection{Immunohistochemistry and Histochemistry}

A Cryotome 2800 Frigocut N (Reichert-Jung, Nussloch, Germany) was used to prepare frozen tissue sections (thickness: $5 \mu \mathrm{m}$ ). The following antibodies were used for immunohistochemical staining according to the manufacturer's protocols: $\mathrm{ED} 1$ for $\mathrm{CD} 68^{+}$monocytes/macrophages, ED2 for $\mathrm{CD}_{163}{ }^{+}$tissue macrophages, R73 for T lymphocytes, OX6 for MHC-II ${ }^{+}$antigen-presenting cells (all antibodies obtained from MorphoSys AbD Serotec GmbH, Duesseldorf, Germany), AD1 for mast cells (BD Biosciences, Heidelberg, Germany) and ANK61 for activated natural killer (NK) cells (Santa Cruz Biotechnology, Heidelberg, Germany). Detection of bound primary antibodies was performed with the Alkaline Phosphatase Anti-Alkaline Phosphatase method (APAAP; DakoCytomation GmbH, Hamburg, Germany), the polyclonal rabbit anti-mouse-immunoglobulin (Z259; Dako DenmarkA/S, Glostrup, Denmark) and the chromogenic substance new fuchsine. 


\subsubsection{Microscopic Equipment}

The stained histological samples were evaluated using a light microscope CX41 (Olympus, Hamburg, Germany) at a visual magnification of $100 \times$. A colour camera DP20 (resolution $1600 \times 1200$ Pixel, CCD size 1/1.8 in; Olympus, Hamburg, Germany) attached via a camera adapter U-TV0.63XC (adapter magnification 0.63×; Olympus, Hamburg, Germany) was used to obtain digital images. Images were taken with an objective magnification of $10 \times$ and represent an area of about $1 \mathrm{~mm}^{2}$ tissue section at the given camera resolution.

\subsubsection{Image Analysis Procedure}

The image analysis program ImageJ version1.44 (U.S. National Institutes of Health, Bethesda, MD, USA) [27] and the software plugins Grid and CellCounter were used to determine the number of positively stained cells in defined areas [28]. Briefly, a grid with a square size of 20,000 pixels was superimposed onto the images, and five representative squares directly adjacent to the implant pockets were examined. If necessary, the area covered by artefact zones and other regions without tissue was measured and deducted from the total analyzed area of 100,000 pixels per image. One pixel corresponded to an area of $0.4796 \mu^{2}$ in the chosen microscopic magnification, based on evaluation of a microscopic slide with a printed length scale. Final results given as positively stained cells per $\mu \mathrm{m}^{2}$ represent the average counts from two independent investigators.

\subsection{Statistical Data Analysis}

Data of copper release measurements are given as mean concentration values \pm standard deviation of analysis. For all morphological analysis, cell count data are given as median cells per $\mu \mathrm{m}^{-2}$ section plane and interquartile range (IQR) with whiskers as minimum and maximum individual cell numbers. The non-parametric Wilcoxon signed rank test was used for pairwise comparison of the number of positively stained cells in the peri-implant tissue on the same experimental day. Results for the same implant type over the course of the three experimental days were analyzed with the non-parametric Kruskal-Wallis test. A $p$-value of less than 0.05 was considered to be statistically significant for all tests. The software system GraphPad Prism version 4.03 (GraphPad Software, Inc., San Diego, CA, USA) was used for statistical analysis.

Acknowledgments: We are grateful to Kirsten Kesselring and Uwe Lindemann for excellent technical support and Marion Quaas for helpful discussions. This study was supported by grants from the federal state of Mecklenburg-Vorpommern (Grant No. UR 04022 10), the Helmholtz Association of German Research Centres (grant no. VH-MV1) and the Federal Ministry of Education and Research (Grant Nos. 13N9779, 13N7796, 13N9775, 13N11183, 13N11188 Campus PlasmaMed).

Author Contributions: B.F., B.N. and M.S. conceived the overall concept of the study. B.F. and M.Po. designed the plasma-physical and XPS experiments, R.B. the copper release measurements, M.S. the in vivo experiments, S.L. the immunohistochemistry and histochemistry experiments, and U.W. the image analysis procedure. B.F. and M.Po. performed the plasma-physical and XPS experiments, C.Z. the copper release measurements, U.W., A.H. and M.Pa. the in vivo experiments, S.L. the immunohistochemistry and histochemistry experiments, and U.W. and S.L. the image analysis procedure. U.W., B.F. and M.S. analyzed the data. U.W., A.H., B.F. and M.S. wrote the paper. G.L., R.B., A.P. and B.N. assisted in the interpretation of data and critically reviewed the manuscript. All authors approved the final manuscript as submitted.

Conflicts of Interest: The authors declare no conflict of interest. The founding sponsors had no role in the design of the study; in the collection, analyses, or interpretation of data; in the writing of the manuscript, and in the decision to publish the results.

\section{References}

1. Esposito, M. Titanium for dental applications. In Titanium in Medicine; Brunette, D.M., Tengvall, P., Textor, M., Thomsen, P., Eds.; Springer: Berlin, Germany, 2001; pp. 182-230.

2. Campoccia, D.; Montanaro, L.; Arciola, C.R. The significance of infection related to orthopedic devices and issues of antibiotic resistance. Biomaterials 2006, 27, 2331-2339. [CrossRef] [PubMed] 
3. Arciola, C.R.; Alvi, F.I.; An, Y.H.; Campoccia, D.; Montanaro, L. Implant infection and infection resistant materials: A mini review. Int. J. Artif. Organs 2005, 28, 1119-1125. [PubMed]

4. Zhao, L.; Chu, P.K.; Zhang, Y.; Wu, Z. Antibacterial coatings on titanium implants. J. Biomed. Mater. Res. B Appl. Biomater. 2009, 91, 470-480. [CrossRef] [PubMed]

5. Schröder, K.; Finke, B.; Polak, M.; Lüthen, F.; Nebe, J.B.; Rychly, J.; Bader, R.; Lukowski, G.; Walschus, U.; Schlosser, M.; et al. Gas-discharge plasma-assisted functionalization of titanium implant surfaces. Mater. Sci. Forum 2010, 638-642, 700-705. [CrossRef]

6. Polak, M.; Ohl, A.; Quaas, M.; Lukowski, G.; Lüthen, F.; Weltmann, K.-D.; Schröder, K. Oxygen and water plasma-immersion ion implantation of copper into titanium for antibacterial surfaces of medical implants. Adv. Eng. Mater. 2010, 12, B511-B518. [CrossRef]

7. Hoene, A.; Prinz, C.; Walschus, U.; Lucke, S.; Patrzyk, M.; Wilhelm, L.; Neumann, H.G.; Schlosser, M. In vivo evaluation of copper release and acute local tissue reactions after implantation of copper-coated titanium implants in rats. Biomed. Mater. 2013, 8, 035009. [CrossRef] [PubMed]

8. Akhavan, B.; Wise, S.; Bilek, M. Substrate-regulated growth of plasma-polymerized films on carbide-forming metals. Langmuir 2016, 32, 10835-10843. [CrossRef] [PubMed]

9. Finke, B.; Luethen, F.; Schroeder, K.; Mueller, P.D.; Bergemann, C.; Frant, M.; Ohl, A.; Nebe, B.J. The effect of positively charged plasma polymerization on initial osteoblastic focal adhesion on titanium surfaces. Biomaterials 2007, 28, 4521-4534. [CrossRef] [PubMed]

10. Hoene, A.; Walschus, U.; Patrzyk, M.; Finke, B.; Lucke, S.; Nebe, B.; Schroeder, K.; Ohl, A.; Schlosser, M. In vivo investigation of the inflammatory response against allylamine plasma polymer coated titanium implants in a rat model. Acta Biomater. 2010, 6, 676-683. [CrossRef] [PubMed]

11. Hoene, A.; Patrzyk, M.; Walschus, U.; Straňák, V.; Hippler, R.; Testrich, H.; Meichsner, J.; Finke, B.; Rebl, H.; Nebe, B.; et al. In vivo examination of the local inflammatory response after implantation of Ti6Al4V samples with a combined low-temperature plasma treatment using pulsed magnetron sputtering of copper and plasma-polymerized ethylenediamine. J. Mater. Sci. Mater. Med. 2013, 24, 761-771. [CrossRef] [PubMed]

12. Xia, Z.; Triffitt, T. A review on macrophage responses to biomaterials. Biomed. Mater. 2006, 1, R1-R9. [CrossRef] [PubMed]

13. Davis, C.; Fischer, J.; Ley, K.; Sarembock, I.J. The role of inflammation in vascular injury and repair. J. Thromb. Haemost. 2003, 1, 1699-1709. [CrossRef] [PubMed]

14. Rodriguez, A.; Voskerician, G.; Meyerson, H.; Macewan, S.R.; Anderson, J.M. T cell subset distributions following primary and secondary implantation at subcutaneous biomaterial implant sites. J. Biomed. Mater. Res. A 2008, 85, 556-565. [CrossRef] [PubMed]

15. Goodman, S.B. Wear particles, periprosthetic osteolysis and the immune system. Biomaterials 2007, 28, 5044-5048. [CrossRef] [PubMed]

16. Tang, L.; Jennings, T.A.; Eaton, J.W. Mast cells mediate acute inflammatory responses to implanted biomaterials. Proc. Natl. Acad. Sci. USA 1998, 95, 8841-8846. [CrossRef] [PubMed]

17. Huss, R.S.; Huddleston, J.I.; Goodman, S.B.; Butcher, E.C.; Zabel, B.A. Synovial tissue-infiltrating natural killer cells in osteoarthritis and periprosthetic inflammation. Arthritis Rheum. 2010, 62, 3799-3805. [CrossRef] [PubMed]

18. Finke, B.; Rebl, H.; Hempel, F.; Schäfer, J.; Liefeith, K.; Weltmann, K.-D.; Nebe, J.B. Aging of plasma-polymerized allylamine nanofilms and the maintenance of their cell adhesion capacity. Langmuir 2014, 30, 13914-13924. [CrossRef] [PubMed]

19. Finke, B.; Polak, M.; Hempel, F.; Rebl, H.; Zietz, C.; Stranak, V.; Lukowski, G.; Hippler, R.; Bader, R.; Nebe, J.B.; et al. Antimicrobial potential of copper-containing titanium surfaces generated by ion implantation and dual high power impulse magnetron sputtering. Adv. Eng. Mater. 2012, 14, B224-B230. [CrossRef]

20. Walschus, U.; Hoene, A.; Neumann, H.-G.; Wilhelm, L.; Lucke, S.; Lüthen, F.; Rychly, J.; Schlosser, M. Morphometric immunohistochemical examination of the inflammatory tissue reaction after implantation of calcium phosphate-coated titanium plates in rats. Acta Biomater. 2009, 5, 776-784. [CrossRef] [PubMed]

21. Kochanowski, A.; Hoene, A.; Patrzyk, M.; Walschus, U.; Finke, B.; Luthringer, B.; Feyerabend, F.; Willumeit, R.; Lucke, S.; Schlosser, M. Examination of the inflammatory response following implantation of titanium plates coated with phospholipids in rats. J. Mater. Sci. Mater. Med. 2011, 22, 1015-1026. [CrossRef] [PubMed] 
22. Wilhelm, L.; Zippel, R.; von Woedtke, T.; Kenk, H.; Hoene, A.; Patrzyk, M.; Schlosser, M. Immune response against polyester implants is influenced by the coating substances. J. Biomed. Mater. Res. A 2007, 83, 104-113. [CrossRef] [PubMed]

23. Schlosser, M.; Zippel, R.; Hoene, A.; Urban, G.; Ueberrueck, T.; Marusch, F.; Koch, A.; Meyer, L.; Wilhelm, L. Antibody response to collagen after functional implantation of different polyester vascular prostheses in pigs. J. Biomed. Mater. Res. A 2005, 72, 317-325. [CrossRef] [PubMed]

24. Schnabelrauch, M.; Wyrwa, R.; Rebl, H.; Bergemann, C.; Finke, B.; Schlosser, M.; Walschus, U.; Lucke, S.; Weltmann, K.-D.; Nebe, J.B. Surface-coated polylactide fiber meshes as tissue engineering matrices with enhanced cell integration properties. Int. J. Polym. Sci. 2014, 6, 439784. [CrossRef]

25. Gristina, A.G. Biomaterial-centered infection: Microbial adhesion versus tissue integration. Science 1987, 237, 1588-1595. [CrossRef] [PubMed]

26. Beamson, G.; Briggs, D. High Resolution XPS of Organic Polymers: The Scienta ESCA 300 Database; Wiley: Chichester, UK; New York, NY, USA, 1992.

27. Gallagher, S.R. Digital Image Processing and Analysis with ImageJ. Curr. Protoc. Essent. Lab. Tech. 2010, 3, A.3C.1-A.3C.24.

28. Walschus, U.; Hoene, A.; Kochanowski, A.; Neukirch, B.; Patrzyk, M.; Wilhelm, L.; Schröder, K.; Schlosser, M. Quantitative immunohistochemical examination of the local cellular reactions following implantation of biomaterials. J. Microsc. 2011, 242, 94-99. [CrossRef] [PubMed]

(C) 2017 by the authors. Licensee MDPI, Basel, Switzerland. This article is an open access article distributed under the terms and conditions of the Creative Commons Attribution (CC BY) license (http:/ / creativecommons.org/licenses/by/4.0/). 